$1997-12-21$

\title{
Statistical Continuum Theory for Inelastic Behavior of a Two- Phase Medium
}

Brent L. Adams

b_I_adams@byu.edu

H. Garmestani

S. Lin

Follow this and additional works at: https://scholarsarchive.byu.edu/facpub

Part of the Mechanical Engineering Commons

Original Publication Citation

Int. J. Plasticity 14: (8) 719-731 (1998)

\section{BYU ScholarsArchive Citation}

Adams, Brent L.; Garmestani, H.; and Lin, S., "Statistical Continuum Theory for Inelastic Behavior of a TwoPhase Medium" (1997). Faculty Publications. 655.

https://scholarsarchive.byu.edu/facpub/655

This Peer-Reviewed Article is brought to you for free and open access by BYU ScholarsArchive. It has been accepted for inclusion in Faculty Publications by an authorized administrator of BYU ScholarsArchive. For more information, please contact ellen_amatangelo@byu.edu. 


\title{
STATISTICAL CONTINUUM THEORY FOR INELASTIC BEHAVIOR OF A TWO-PHASE MEDIUM
}

\author{
H. Garmestani, ${ }^{1}$ S. Lin ${ }^{1}$ and B. L. Adams ${ }^{2}$ \\ ${ }^{1}$ Mechanical Engineering at the FAMU-HU College of Engineering and Center for Materials Research and \\ Technology (MARTECH), Tallahassee, FL 32310, U.S.A. \\ ${ }^{2}$ Carnegie Mellon University, Pittsburg, PA 15213-3890, U.S.A.
}

http://www.sciencedirect.com/science/journal/07496419

\begin{abstract}
A statistical continuum mechanics formulation is presented to predict the inelastic behavior of a medium consisting of two isotropic phases. The phase distribution and morphology are represented by a two-point probability function. The isotropic behavior of the single phase medium is represented by a power law relationship between the strain rate and the resolved local shear stress. It is assumed that the elastic contribution to deformation is negligible. A Green's function solution to the equations of stress equilibrium is used to obtain the constitutive law for the heterogeneous medium. This relationship links the local velocity gradient to the macroscopic velocity gradient and local viscoplastic modulus. The statistical continuum theory is introduced into the localization relation to obtain a closed form solution. Using a Taylor series expansion an approximate solution is obtained and compared to the Taylor's upper-bound for the inelastic effective modulus. The model is applied for the two classical cases of spherical and unidirectional discontinuous fiber-reinforced two-phase media with varying size and orientation.
\end{abstract}

\section{INTRODUCTION}

Voigt (1889) and Reuss (1929) proposed two methods to calculate the effective elastic moduli of polycrystals. The first takes the average of the elastic moduli of the crystallites over all orientations as effective moduli of the polycrystal, whereas the second uses the average of the elastic compliances. Hill (1952) proved that the two results are the upper and the lower bounds to the true effective elastic moduli of the polycrystal. Adding the additional information that the medium is macroisotropic and the elastic moduli of neighboring grains are uncorrelated, Hashin and Shtrikman (1962) found narrower bounds which is classified as 2nd order bounds, whereas the bounds of Voigt and Reuss are 1st order bounds. Taylor (1938) set up a uniform strain model for large deformation polycrystals. The objective was to estimate the effective moduli of a polycrystalline solid from single crystallites.

Models based upon Taylor's assumption of uniform plastic strain (or strain rate), similar to Voigt's, together with the modern texture theory, have been used to predict the

*Corresponding author. 
mechanical anisotropy of polycrystals for several decades now. Comparisons between the simulations of these models and the measured texture evolution have also exhibited firstorder agreement (Hill, 1965; Beran, 1968; Kroner, 1972, 1987). Corrections to Taylor's upper-bound were provided in different forms based on Hill's self-consistent model (Bishop and Hill, 1951; Hill, 1965, 1967; Hutchinson, 1976). The objective was to estimate the effective moduli of composites or polycrystalline solids from the mechanical behavior of their constituents. In such modeling efforts a specific morphology and phase distribution was assumed for the constituents.

Kroner (1987) developed a model to investigate the elastic properties of polycrystalline materials under small deformation using statistical continuum mechanics analysis. Adams et al. (1989) applied this theory to viscoplastic deformation of heterogeneous polycrystalline materials. These new models take into account the heterogeneity of strain (or strain rate) in the microstructure. Microstructure can be defined as the spatial distribution of phases, particles, and crystalline orientation. The traditional methods of representation of heterogeneity in microstructures concentrated on the formulation of anisotropy (or texture). In the case of multi-phase media an average based on the volume fractions of the different phases have usually been used as an approximation to the solution by assuming independent texture evolution for each phase. For polycrystalline materials, Orientation Distribution Functions (ODF), which are used to quantify texture, are found inadequate for such representation. The statistical model for large deformation of polycrystalline materials requires that the information for the microstructure not be limited to lattice orientations and also include the spatial correlations. The spatial correlations are referred to as the orientation coherence functions (OCF) (Adams et al., 1989). The statistical viewpoint takes OCFs to be $n$-point probability density functions, which describe the correlation of lattice orientations between points in the polycrystal separated by specified vectors. Since each single orientation represents a distinct level of heterogeneity, the structure can be assumed to contain a number of states of orientations. The characterization of such a complex structure based on statistical formulation focused on structural changes and as a result limited further understanding of probability functions and their limitation to predict effective properties (Beran et al., 1996). To explore this further and concentrate on the property of probability functions, a two phase medium is chosen for exploration and it is assumed that each phase is isotropic. A two point probability density function is used to represent the spatial correlation. In a study of two phase media by Corson $(1974 a, b)$, it was found that an exponential form can adequately represent the two point probability density function for most microstructures which exhibit a global isotropy. An anisotropic version of such a formulation is adopted in this paper to represent the probability functions. Such a procedure is important since the simulation of the continuum mechanics theory requires a closed form for the probability function which can correctly represent the microstructure. This can also increase the efficiency and speed of the numerical simulation. Molinari et al. (1987) used a two-Green's-function solution in a self-consistent scheme to analyze the large deformation polycrystal viscoplasticity. They used the tangent-modulus formulation in their self-consistent approach to predict texture evolution. In the present paper a secant modulus version of that formulation for the isotropic constitutive law is selected to describe the behavior of each phase. The Green's function solution to the field equations proposed by Molinari et al. (1987) is a first step of deriving the final closed form solution to the statistical theory. 


\section{SINGLE PHASE ISOTROPIC BEHAVIOR}

Following Hutchinson (1976), a power-law steady-state creep law is chosen for each phase. The isotropic constitutive law (elastic-rates are neglected) is defined independently for each phase:

$$
\frac{D}{D^{*}}=\left(\frac{\sigma}{\sigma^{*}}\right)^{n}
$$

where $D^{*}$ and $\sigma^{*}$ are the strain rate and stress invariants for the reference configuration, $n$ is the inverse strain rate sensitivity parameter $(>>1)$. The invariants of the strain-rate tensor $D$ and the deviatoric stress tensor $\mathrm{s}$ are defined by

$$
\begin{gathered}
D=\left(\frac{2}{3} D_{j i} D_{j i}\right)^{1 / 2} \\
\sigma=\left(\frac{3}{2} S_{i j} S_{i j}\right)^{1 / 2} \\
D_{i j}=\left[\frac{3}{2} \frac{D}{\sigma}\right] I_{i j k l} S_{k l}=\left[\frac{3}{2} \frac{D^{*}}{\sigma^{*}}\left(\frac{\sigma}{\sigma^{*}}\right)^{n-1}\right] I_{i j k l} S_{k l}=M_{i j k l} S_{k l} \\
S_{i j}=\left[\frac{2}{3} \frac{\sigma}{D}\right] I_{i j k l} D_{k l}=\left[\frac{2}{3} \frac{\sigma^{*}}{D^{*}}\left(\frac{D}{D^{*}}\right)^{\frac{1-n}{n}}\right] I_{i j k l} D_{k l}=N_{i j k l} D_{k l} \\
I_{i j k l}=\frac{1}{2}\left(\delta_{i k} \delta_{j l}+\delta_{i l} \delta_{j k}\right) \\
N_{i j k l}=N_{j i k l}=N_{i j l k}=N_{k l i j}
\end{gathered}
$$

where, $M=[N]^{-1}$ and it contains 4 th rank symmetry as in eqn (7), $I$ is the isotropic fourth rank tensor. Equations (4) and (5) can also be described in the short notation:

$$
\begin{aligned}
& \mathbf{D}=\mathbf{M S} \\
& \mathbf{S}=\mathbf{N D} .
\end{aligned}
$$

The secant modulus is defined as the slope of the stress-strain rate curve at a certain strain rate level as shown in Fig. 1. A full Cauchy stress tensor is the summation of the deviatoric stress and the hydrostatic pressure:

$$
T_{i j}=N_{i j k l} D_{k l}-p \delta_{i j}
$$




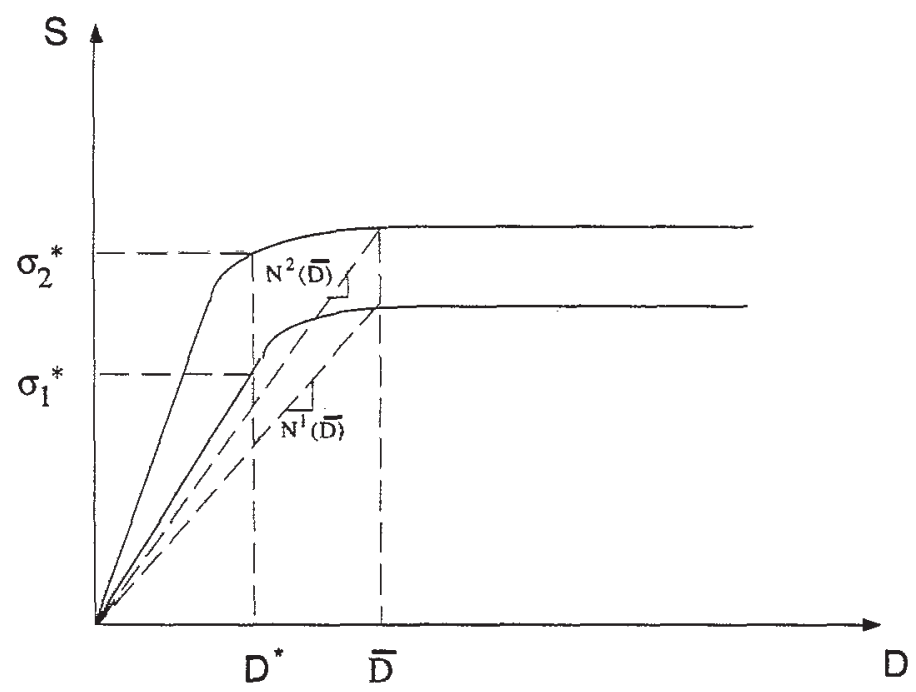

Fig. 1. Definition of the secant modulus for the two isotropic viscoplastic phases.

\section{GREEN'S FUNCTION SOLUTIONS: THE INTERACTION LAW}

The local behavior has been determined for each phase according to eqn (8). A macroscopic homogeneous velocity gradient $\bar{L}_{i j}=\bar{v}_{i, j}$ and a macroscopic pressure $\bar{p}$ are imposed on the medium at infinity. It is intended to find the local velocity gradient $L_{i j}=v_{i j}$. The velocity gradient tensor $L_{i j}$ is a sum of the symmetrical part, strain-rate tensor $D_{k l}$ and the antisymmetrical part, rate-of-rotation tensor $\omega_{k l}$. Due to the symmetry properties of the inelastic modulus $N_{i j k l}$ given by eqn (7), $N_{i j k l} \omega_{k l}=0$ and therefore:

$$
N_{i j k l} L_{k l}=N_{i j k l}\left(D_{k l}+\omega_{k l}\right)=N_{i j k l} D_{k l} .
$$

Locally, equilibrium is required throughout the medium. From eqn (10):

$$
T_{i j, j}=\left(N_{i j k l} D_{k l}\right)_{, \hat{j}}-p_{, i}=\left(N_{i j k l} L_{k l}\right)_{j}-p_{, i}=0
$$

Let us separate $N_{i j k l}$ into a uniform part $N_{i j k l}^{0}$ and a spatially dependent part $\tilde{N}_{i j k l}$ :

$$
N_{i j k l}=N_{i j k l}^{0}+\tilde{N}_{i j k l}
$$

$N_{i j k}^{0}$ is defined as:

$$
\left.N_{i j k}^{0}=\int f h\right) N_{i j k l}(\bar{L}, h) \mathrm{d} h
$$

where $h$ is a set of state variables and $f(h)$ is the probability density for the occurrence of state $h$ in the medium. For a two-isotropic-phase media, $h$ represents phase 1 and 2, thus $f(h)$ is the corresponding volume fraction. As a result: 


$$
\begin{gathered}
N_{i j k l}^{0}=f_{1} N_{i j k l}^{1}(\bar{L})+f_{2} N_{i j k l}^{2}(\bar{L}) \\
f_{1}+f_{2}=1
\end{gathered}
$$

$N_{i j k l}^{1}(\bar{L})$ and $N_{i j k l}^{2}(\bar{L})$ represent the secant moduli of phase 1 and phase 2 , respectively, at the velocity gradient level of $\bar{L}$.

Substituting eqn (13) into the eqn (12):

$$
\begin{gathered}
N_{i j k l}^{0} L_{k l, j}-p_{, i}+F_{i}=0 \\
F_{i}=\left[\tilde{N}_{i j k l} L_{k l}\right]_{, j}
\end{gathered}
$$

where $F_{i}$ can be considered as a "fictitious" body force. The incompressibility condition dictates:

$$
L_{i i}=0 \text {. }
$$

Equations (17) and (19) represent a set of four independent equations to be solved for four unknowns $v_{i}$ and $p$ (velocity and pressure). This system of equations can be solved by the Green's function method. The medium is assumed to be infinite. The Green's functions in the infinite space are $G_{i j}\left(\mathbf{r}-\mathbf{r}^{\prime}\right)$ and $H_{k}\left(\mathbf{r}-\mathbf{r}^{\prime}\right)$, where $\mathbf{r}$ and $\mathbf{r}^{\prime}$ represent points in space. At infinity, $\left|\mathbf{r}-\mathbf{r}^{\prime}\right| \rightarrow \infty, G_{i j}$ and $H_{k}$ should approach zero. The $G_{i j}$ and $H_{k}$ are solutions to the equations following eqns (20) and (21) which are derived from eqns (17) and (19) as:

$$
\begin{gathered}
N_{i j k l}^{0} G_{k m, l j}\left(\mathbf{r}-\mathbf{r}^{\prime}\right)-H_{m, i}\left(\mathbf{r}-\mathbf{r}^{\prime}\right)+\delta_{i m} \delta\left(\mathbf{r}-\mathbf{r}^{\prime}\right)=0 \\
G_{k m, k}\left(\mathbf{r}-\mathbf{r}^{\prime}\right)=0 .
\end{gathered}
$$

The function $\delta\left(\mathbf{r}-\mathbf{r}^{\prime}\right)$ is the Dirac function located at $\mathbf{r}^{\prime}$ the term $\delta_{i m} \delta\left(\mathbf{r}-\mathbf{r}^{\prime}\right)$ represents the $i$ th component of a unit force acting at $\mathbf{r}^{\prime}$ and being parallel to the direction $m$ for a fixed $m$. Then the solutions to the eqns (17) and (19) are represented by:

$$
\begin{aligned}
& v_{i}(\mathbf{r})=\bar{\nu}_{i}+\int_{\mathbf{r}^{\prime} \in V} G_{i j}\left(\mathbf{r}-\mathbf{r}^{\prime}\right) F_{j}\left(\mathbf{r}^{\prime}\right) \mathrm{d} \mathbf{r}^{\prime} \\
& p(\mathbf{r})=\bar{p}+\int_{\mathbf{r}^{\prime} \in V} H_{i}\left(\mathbf{r}-\mathbf{r}^{\prime}\right) F_{i}\left(\mathbf{r}^{\prime}\right) \mathrm{d} \mathbf{r}^{\prime} .
\end{aligned}
$$

Finally, we expect a closed form solution for the local velocity gradient $L_{i j}=v_{i, j}$. After differentiating the two sides of eqn (22), we obtain the interaction law:

$$
\begin{aligned}
L_{i k}(\mathbf{r}) & =\bar{L}_{i k}+\int_{r^{\prime} \in V} G_{i j, k}\left(\mathbf{r}-\mathbf{r}^{\prime}\right)\left[\tilde{N}_{j l r s}\left(L\left(\mathbf{r}^{\prime}\right), h\left(\mathbf{r}^{\prime}\right)\right) L_{r s}\left(\mathbf{r}^{\prime}\right)\right]_{l} \mathrm{~d} \mathbf{r}^{\prime} \\
& =\bar{L}_{i k}+\int_{r^{\prime} \in V} G_{i j, k l}\left(\mathbf{r}-\mathbf{r}^{\prime}\right) \tilde{N}_{j l r s}\left(L\left(\mathbf{r}^{\prime}\right), h\left(\mathbf{r}^{\prime}\right)\right) L_{r s}\left(\mathbf{r}^{\prime}\right) \mathrm{d} \mathbf{r}^{\prime}
\end{aligned}
$$


The physical meaning of the interaction law is that the local velocity gradient at $\mathbf{r}$ differs from the imposed macroscopic velocity gradient by the convolution over the infinite volume $V$ of a polarized deviatoric stress, $\tilde{\sigma}$, which depends on the local velocity gradient and the state at $\mathbf{r}^{\prime} \in V$. This polarized stress is defined as:

$$
\tilde{\sigma}_{j l}\left(\mathbf{r}^{\prime}\right)=\tilde{N}_{j l r s}\left(L\left(\mathbf{r}^{\prime}\right), h\left(\mathbf{r}^{\prime}\right)\right) L_{r s}\left(\mathbf{r}^{\prime}\right)
$$

It represents the effect on material point $\mathbf{r}$ by the difference between the local velocity gradient at $\mathbf{r}^{\prime}$ and the imposed macroscopic uniform velocity gradient. $G_{i j, k l}\left(\mathbf{r}-\mathbf{r}^{\prime}\right) \tilde{N}_{j i r s}\left(L\left(\mathbf{r}^{\prime}\right), h\left(\mathbf{r}^{\prime}\right)\right) L_{r s}\left(\mathbf{r}^{\prime}\right)$ represents the total outcome. $G_{i j, k l}\left(\mathbf{r}^{\prime}-\mathbf{r}^{\prime}\right)$ has a singular value at $\mathbf{r}=\mathbf{r}^{\prime}$. A proper solution to this problem is to construct solutions for a finite small volume $V_{\mathrm{c}}$ surrounding point $\mathbf{r}$, then calculate the average value of velocity gradient to replace the value at $\mathbf{r}$ (Kroner, 1987). In the final numerical calculation, a very small volume compared to the whole specimen is used in order to obtain a solution. This small volume is chosen to be one percent of the average size of the second phase as shown later. Let $L^{0}(\mathbf{r})$ be this averaged value:

$$
L_{i k}^{0}=\bar{L}_{i k}+\frac{1}{V_{c}} \int_{r \in V_{c}} \int_{r^{\prime} \in V} G_{i j, k l}\left(\mathbf{r}-\mathbf{r}^{\prime}\right) \tilde{N}_{j l r s}\left(L\left(\mathbf{r}^{\prime}\right), h\left(\mathbf{r}^{\prime}\right)\right) L_{r s}\left(\mathbf{r}^{\prime}\right) \mathrm{d} \mathbf{r}^{\prime} \mathrm{d} \mathbf{r}
$$

For eqn (26), there is an abbreviated notation:

$$
\mathbf{L}^{0}(\mathbf{r})=\overline{\mathbf{L}}+\mathbf{G}\left(\mathbf{r}-\mathbf{r}^{\prime}\right)^{*} \mathbf{N}\left(\mathbf{r}^{\prime}\right) \mathbf{L}^{0}\left(\mathbf{r}^{\prime}\right)
$$

The symmetrical part of eqn (27) defines the local strain-rate tensor

$$
\mathbf{D}^{0}(\mathbf{r})=\overline{\mathbf{D}}+\Gamma\left(\mathbf{r}-\mathbf{r}^{\prime}\right)^{*} \mathbf{N}\left(\mathbf{r}^{\prime}\right) \mathbf{L}^{0}\left(\mathbf{r}^{\prime}\right)
$$

where

$$
\Gamma_{i j k l}=\frac{1}{4}\left(G_{j k, i l}+G_{i k, j l}+G_{j l, i k}+G_{i l, j k}\right)
$$

The antisymmetrical part defines the total spin tensor

$$
\mathbf{w}^{0}(\mathbf{r})=\overline{\mathbf{w}}+\Lambda\left(\mathbf{r}-\mathbf{r}^{\prime}\right)^{*} \mathbf{N}\left(\mathbf{r}^{\prime}\right) \mathbf{L}^{0}\left(\mathbf{r}^{\prime}\right)
$$

where

$$
\Lambda_{i j k l}=\frac{1}{4}\left(G_{j k, i l}-G_{i k, j l}+G_{j l, i k}-G_{i l, j k}\right)
$$

To solve the Green's functions $G_{i j}$ and $H_{k}$, a Fourier transformation of eqns (20) and (21) is used:

$$
\begin{gathered}
-N_{i j k l}^{0} k_{j} k_{l} \tilde{G}_{k m}(\mathbf{k})+i k_{i} \tilde{H}_{m}(\mathbf{k})+\delta_{i m}=0 \\
k_{k} \tilde{G}_{k m}(\mathbf{k})=0 .
\end{gathered}
$$


A linear set of equations are derived which are solved for $\tilde{G}_{k m}(\mathbf{k})$ and $\tilde{H}_{m}(\mathbf{k})$. Using the property of the inverse Fourier transform:

$$
\begin{aligned}
G_{i j, k l}\left(\mathbf{r}-\mathbf{r}^{\prime}\right) & =-\frac{1}{8 \pi^{3}} \int_{\mathbf{k} \in k^{3}} k_{k} k_{l} \tilde{G}_{i j}(\mathbf{k}) e^{-i \mathbf{k}\left(\mathbf{r}-\mathbf{r}^{\prime}\right)} \mathrm{d} k^{3} \\
H_{m, i}\left(\mathbf{r}-\mathbf{r}^{\prime}\right) & =-\frac{i}{8 \pi^{3}} \int_{\mathbf{k} \in k^{3}} k_{i} \tilde{H}_{m}(\mathbf{k}) e^{-i \bar{k}\left(\mathbf{r}-\mathbf{r}^{\prime}\right)} \mathrm{d} k^{3} .
\end{aligned}
$$

The solution for this problem is already given for an isotropic incompressible medium (Molinari et al., 1987).

$$
N_{i j k l}^{0}=\frac{\mu}{2}\left(\delta_{i j k} \delta_{j l}+\delta_{i l} \delta_{j k}\right)=\mu I_{i j k l}
$$

where $\mu$ depends on the properties of phase 1 , phase 2 and their volume fractions. $\mu=2\left(v_{1}\left[\frac{3}{2} \frac{D_{1}}{\sigma_{1}}\right]+v_{2}\left[\frac{3}{2} \frac{D_{2}}{\sigma_{2}}\right]\right)$. Here $v_{1}, v_{2}$ are volume fractions, $D_{1}, D_{2}, \sigma_{1}, \sigma_{2}$ are described in eqns (2) and (3).

Then:

$$
\begin{gathered}
\tilde{G}_{n i}(k)=\frac{2}{\mu k^{2}} \delta_{n i}-\frac{2}{\mu k^{4}} k_{n} k_{i} \\
\tilde{H}_{m}(\mathbf{k})=\frac{\sqrt{-1}}{k^{2}} k_{m} .
\end{gathered}
$$

In later sections, a methodology is presented for the numerical simulation of the results. In the following the evaluation of the convolution integrals $\mathbf{G}\left(\mathbf{r}-\mathbf{r}^{\prime}\right) * \mathbf{N}\left(\mathbf{r}^{\prime}\right) \mathbf{L}^{0}\left(\mathbf{r}^{\prime}\right)$ is described. First, the medium is divided into a number of small cubes of volume $\Delta^{3}$ shown as $V_{\mathrm{c}}$ and $V_{\mathrm{c}}^{\prime}$ in Fig. 2. For a fixed $\mathbf{r}$ at $V_{\mathrm{c}}$, we consider a second cube of volume $V_{\mathrm{c}}^{\prime}$ which contains $\mathbf{r}^{\prime}$. The full integral around $\mathbf{r}^{\prime} \in V$ can be taken as the summation of all cubes $V_{c}^{\prime} \in V$ except $V_{\mathrm{c}}$. Figure 2 shows two cubes $V_{\mathrm{c}}$ and $V_{\mathrm{c}}^{\prime}$, where $\mathbf{c}$ and $\mathbf{c}^{\prime}$ represent the center positions of each cube. Additional details for the calculation of $G$ and $H$ is presented elsewhere (Ahzi, 1987).

$$
\begin{aligned}
\mathbf{G}\left(\mathbf{r}-\mathbf{r}^{\prime}\right) * \tilde{\mathbf{N}}\left(\mathbf{r}^{\prime}\right) \mathbf{L}^{0}\left(\mathbf{r}^{\prime}\right) & =\frac{1}{V_{c}} \int_{\mathbf{r} \in V_{c}} \int_{\mathbf{r}^{\prime} \in V} G_{i j, k l}\left(\mathbf{r}-\mathbf{r}^{\prime}\right) F_{j l}\left(\mathbf{r}^{\prime}\right) \mathrm{d} \mathbf{r}^{\prime} \mathrm{d} \mathbf{r} \\
& =\frac{1}{V_{c}} \int_{\mathbf{r} \in V_{c}}\left\{\sum_{V_{c}^{\prime} \in V}\left[\int_{\mathbf{r}^{\prime} \in V_{c}^{\prime}} G_{i j, k l}\left(\mathbf{r}-\mathbf{r}^{\prime}\right) F_{j l}\left(\mathbf{r}^{\prime}\right) \mathrm{d} \mathbf{r}^{\prime}\right]\right\} \mathrm{d} \mathbf{r} \\
& =\sum_{V_{c} \in V}\left\{\frac{1}{V_{c}} \int_{\mathbf{r} \in V_{c}} \int_{\mathbf{r}^{\prime} \in V_{c}} G_{i j, k l}\left(\mathbf{r}-\mathbf{r}^{\prime}\right) F_{j l}\left(\mathbf{r}^{\prime}\right) \mathrm{d} \mathbf{r}^{\prime} \mathrm{d} \mathbf{r}\right\} .
\end{aligned}
$$

Now, $F_{j l}\left(\mathbf{r}^{\prime}\right)=\mathbf{N}\left(\mathbf{r}^{\prime}\right) \mathbf{L}^{0}\left(\mathbf{r}^{\prime}\right)$ is taken to be constant over $V_{c}^{\prime}$. Applying the inverse Fourier transform of $\tilde{G}_{n i}$, the final solution for eqn (39) is obtained by using eqn (40). The result is 


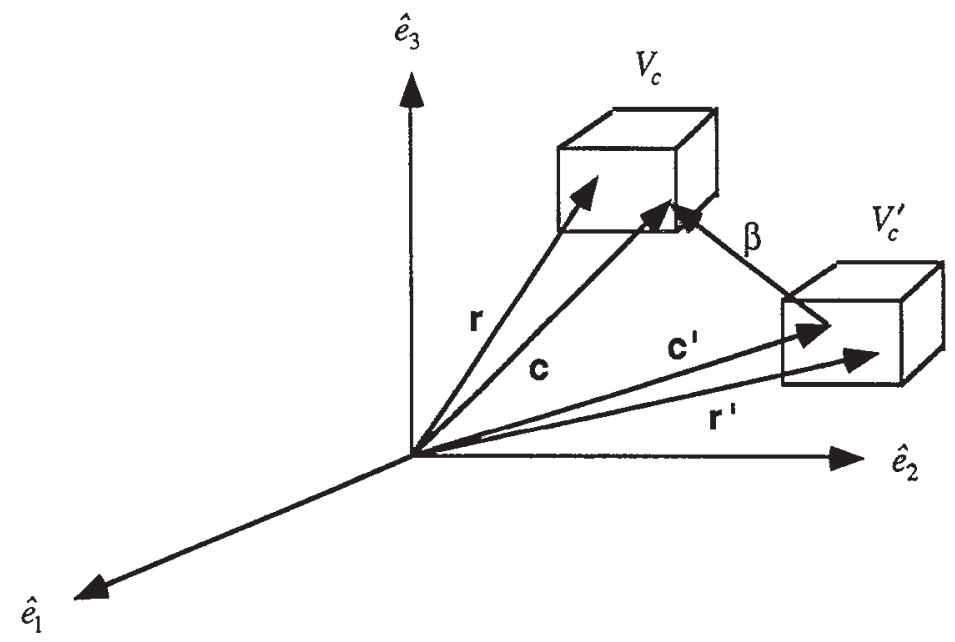

Fig. 2. Two elements geometry of the convolution integral problem $\mathrm{G} * \mathrm{~F}$.

presented as an integral solution over infinite region followed by some differential process (Morris, 1970; Muram, 1987).

$$
\frac{1}{V_{c}} \int_{\mathbf{r} \in V_{c}} \int_{\mathbf{r} \in V_{c}} G_{i j, k l}\left(\mathbf{r}-\mathbf{r}^{\prime}\right) F_{j l}\left(\mathbf{r}^{\prime}\right) \mathrm{d} \mathbf{r}^{\prime} \mathrm{d} \mathbf{r}=\frac{F_{j l}}{\bar{V}_{c}} \int_{\mathbf{r} \in V_{c}} \int_{\mathbf{r}^{\prime} \in V_{c}^{\prime}} G_{i j, k l}\left(\mathbf{r}-\mathbf{r}^{\prime}\right) \mathrm{d} \mathbf{r}^{\prime} \mathrm{d} \mathbf{r} .
$$

\section{PROBABILITY FUNCTION AND STATISTICAL FORMULATIONS}

Equation (27) can be written as a Taylor series expansion about $\bar{L}$ (assuming $\left.L^{0}-\bar{L} \ll L^{0}\right)$. Keeping only the first few terms, we get:

$$
\begin{gathered}
\left.\mathbf{L}^{0}=\overline{\mathbf{L}}+\mathbf{G} *\left(\tilde{\sigma}(\overline{\mathbf{L}})+\tilde{\sigma}^{\prime}(\overline{\mathbf{L}}) \tilde{\mathbf{L}}^{0}+\frac{1}{2 !} \tilde{\sigma}^{\prime \prime}(\overline{\mathbf{L}})\left(\tilde{\mathbf{L}}^{0}\right)^{2}+\geq\right)\left(\tilde{\mathbf{L}}^{0}\right)^{3}\right) \\
\tilde{\mathbf{L}}^{0}=\mathbf{L}^{0}-\overline{\mathbf{L}} .
\end{gathered}
$$

The zeroth-order term, $\mathbf{L}^{0}=\mathbf{L}$ is called the Taylor term (Taylor, 1938). Here only the first-order correction to the homogeneous Taylor solution is considered. In the final solution, only the first order correction to the Taylor's term is calculated. The second order correction was calculated by using the iterative method and was found to be negligible. Then eqn (41) becomes:

$$
\mathbf{L}^{0}(\mathbf{r})=\overline{\mathbf{L}}+\mathbf{G} * \tilde{\sigma}(\overline{\mathbf{L}})=\overline{\mathbf{L}}+\mathbf{G}\left(\mathbf{r}-\mathbf{r}^{\prime}\right) * \tilde{\mathbf{N}}\left(\overline{\mathbf{L}}, h\left(\mathbf{r}^{\prime}\right)\right) \overline{\mathbf{L}}
$$

In the following the statistical concept is introduced with proper correlation functions to relate the local to global properties. Let us consider the ensemble average of the local 
velocity gradients for many particles belonging to the same state $h$. Each particle is surrounded by a structure which is different from all other particles even if they are at the same state. This results in a distinctive velocity gradient for each particle. Here we neglect the difference of the local velocity gradient belonging to the particles with the same state and assume that all these particles have one local velocity gradient value which is calculated from the ensemble average. Symbol $<>_{h}$ denotes the ensemble average over particles at state $h$.

$$
\left\langle()_{i}\right\rangle=\frac{1}{N} \sum_{i=1}^{N}()_{i} .
$$

Combining the ensemble average with eqn (40), we get:

$$
\left\langle\mathbf{L}^{0}(\mathbf{r})\right\rangle_{h}=\overline{\mathbf{L}}+\mathbf{G}\left(\mathbf{r}-\mathbf{r}^{\prime}\right) *\left\langle\tilde{\sigma}\left(\overline{\mathbf{L}}, h\left(\mathbf{r}^{\prime}\right)\right)\right\rangle_{h}=\overline{\mathbf{L}}+\mathbf{G}\left(\mathbf{r}-\mathbf{r}^{\prime}\right) *\left\langle\tilde{\mathbf{N}}\left(\mathbf{L}, h\left(\mathbf{r}^{\prime}\right)\right)\right\rangle_{h} \mathbf{L}
$$

which represents the first-order statistical localization law. The ensemble average of the space dependent modulus $\left\langle\tilde{\mathbf{N}}\left(\mathbf{L}, h\left(\mathbf{r}^{\prime}\right)\right)\right\rangle_{h}$ can be described in terms of the conditional 2point probability density function of state.

$$
\langle\tilde{\mathbf{N}}(\mathbf{L}, h)\rangle_{h_{n}}=\int f\left(\mathbf{r}^{\prime} \in h \mid \mathbf{r} \in h_{n}\right) \tilde{\mathbf{N}}(\mathbf{L}, h) \mathrm{d} h
$$

$f\left(\mathbf{r}^{\prime} \in h \mid \mathbf{r} \in h_{n}\right)$ means the probability that $\mathbf{r}^{\prime}$ belongs to state $h$ given that $\mathbf{r}$ is at state $h_{n}$. In this paper, $h$ has two values: $h_{1}$ and $h_{2}$, representing isotropic phase 1 and phase 2 , respectively, then:

$$
\begin{gathered}
\langle\tilde{\mathbf{N}}(\mathbf{L}, h)\rangle_{h_{1}}=f\left(\mathbf{r}^{\prime} \in h_{1} \mid \mathbf{r} \in h_{1}\right) \tilde{N}^{1}(\overline{\mathbf{L}})+f\left(\mathbf{r}^{\prime} \in h_{2} \mid \mathbf{r} \in h_{1}\right) \tilde{N}^{2}(\overline{\mathbf{L}}) \\
\langle\tilde{\mathbf{N}}(\mathbf{L}, h)\rangle_{h_{2}}=f\left(\mathbf{r}^{\prime} \in h_{1} \mid \mathbf{r} \in h_{2}\right) \tilde{N}^{1}(\overline{\mathbf{L}})+f\left(\mathbf{r}^{\prime} \in h_{2} \mid \mathbf{r} \in h_{2}\right) \tilde{N}^{2}(\overline{\mathbf{L}}) \\
\left\langle\mathbf{L}^{0}(\mathbf{r})\right\rangle_{h_{1}}=\overline{\mathbf{L}}+\mathbf{G}+\left(\mathbf{r}-\mathbf{r}^{\prime}\right) *\left\langle\tilde{\mathbf{N}}\left(\overline{\mathbf{L}}, h\left(\mathbf{r}^{\prime}\right)\right)\right\rangle_{h_{1}} \overline{\mathbf{L}} \\
\left\langle\mathbf{L}^{0}(\mathbf{r})\right\rangle_{h_{2}}=\overline{\mathbf{L}}+\mathbf{G}+\left(\mathbf{r}-\mathbf{r}^{\prime}\right) *\left\langle\tilde{\mathbf{N}}\left(\overline{\mathbf{L}}, h\left(\mathbf{r}^{\prime}\right)\right)\right\rangle_{h_{2}} \overline{\mathbf{L}}
\end{gathered}
$$

It is easy to note that the solution to this problem lies in the existence of the proper probability function for a specific medium. For a two-isotropic-phase medium, we can use the functional form presented by Corson $(1974 a, b)$ (Miller, 1969).

$$
P_{i j}(r)=\alpha^{i j}+\beta_{i j} \exp \left(-c_{i j} r^{n_{i j}}\right)
$$

$i=1,2 ; j=1,2 ; P_{i j}(r)$ means the probability occurrence of one point in phase $i$ and the other point which is located a distance $(r)$ away in phase $j$. It is easy to see that the equation is orientation independent or $P_{\mathrm{ij}}$ is only a function of the magnitude of $\mathbf{r}-\mathbf{r}^{\prime}$ as 
shown in eqn (50), $\alpha_{i j}$ and $\beta_{i j}$ depend on the volume fractions $V_{1}, V_{2}$ of the two phases (Table 1). $c_{\mathrm{ij}}$, and $n_{\mathrm{ij}}$ are empirical constants determined by a least squares fit for the measured data and the functional form chosen for $P_{\mathrm{ij}}$.

$f$ and $P_{\mathrm{ij}}$ are different: $f$ is defined as a conditional probability, whereas $P_{\mathrm{ij}}$ is absolute probability. The relationship between $f$ and $P_{\mathrm{ij}}$ are presented as:

$$
\begin{array}{ll}
f\left(\mathbf{r}^{\prime} \in h_{1} \mid \mathbf{r} \in h_{1}\right)=P_{11} / V_{1} & f\left(\mathbf{r}^{\prime} \in h_{2} \mid \mathbf{r} \in h_{1}\right)=P_{12} / V_{1} \\
f\left(\mathbf{r}^{\prime} \in h_{1} \mid \mathbf{r} \in h_{2}\right)=P_{21} / V_{2} & f\left(\mathbf{r}^{\prime} \in h_{2} \mid \mathbf{r} \in h_{2}\right)=P_{22} / V_{2} .
\end{array}
$$

Macroscopic deviatoric Cauchy stress $\Sigma$ is related to macroscopic velocity gradient $\mathbf{L}$ by substituting eqns (48) and (49) into eqn (52). This relationship is called macroscopic constitutive law.

$$
\Sigma=\int f(h)\left\langle\sigma_{i j}\right\rangle_{h} \mathrm{~d} h=\int f(h) N_{i j k l}(\mathbf{L}, h)\left\langle L_{k l}^{0}\right\rangle_{h} \mathrm{~d} h=V_{1} N_{i j k l}^{1}\left\langle L_{k l}^{0}\right\rangle_{h_{1}}+V_{2} N_{i j k l}^{2}\left\langle L_{k l}^{0}\right\rangle_{h_{2}}
$$

\section{RESULTS AND SIMULATION}

The statistical theory is applied to a two-phase medium with both phases assumed isotropic. The medium is divided into a number of small identical cubic elements. The descritization process follows the general rule that that there should be enough number of elements within each region of investigation so that the final structure can produce a statistical representation of the microstructure. In this analysis the cubic specimen with side length $l$ was divided into $n \times n \times n$ smaller cubes such that $n=c \times l / g$, where $g$ is the average representative size of the inclusions or the second phase. The constant $c$ was determined by trial and error to minimize the error. In this process a value $c=100$ is chosen. It is assumed that each element represents one microstructural state (one phase). Imposing a macroscopic velocity gradient $\mathbf{L}$, it is intended to calculate the velocity gradient in particles of state $h_{1}$, the velocity gradient in particles of state $h_{2}$ and macroscopic deviatoric Cauchy stress $\Sigma$.

Two computer generated microstructures are used for the purpose of this study. The first microstructure (Fig. 3. microstructure a) is generated by allocating centers of circles of radii $r$ at random location until a specific volume fraction is obtained. The second microstructure (Fig. 3. microstructure b) is produced by allocating centers of equivalent

\begin{tabular}{|c|c|c|c|c|}
\hline \multirow[b]{2}{*}{$P_{i j}$} & \multicolumn{2}{|c|}{ Boundary conditions } & \multicolumn{2}{|c|}{ Resultant coefficients } \\
\hline & $r=0$ & $r \rightarrow \infty$ & $\alpha_{i j}=$ & $\beta_{i j}=$ \\
\hline $\begin{array}{l}P_{11} \\
P_{12} \\
P_{21} \\
P_{22}\end{array}$ & $\begin{array}{r}V_{1} \\
0 \\
0 \\
V_{2}\end{array}$ & $\begin{array}{r}V_{1}^{2} \\
V_{1} V_{2} \\
V_{1} V_{2} \\
V_{2}^{2}\end{array}$ & $\begin{array}{l}V_{1}^{2} \\
V_{1}^{2} \\
V_{1}^{2} \\
V_{2}^{2}\end{array}$ & $\begin{array}{r}V_{1} V_{2} \\
-V_{1} V_{2} \\
-V_{1} V_{2} \\
V_{1} V_{2}\end{array}$ \\
\hline
\end{tabular}
narrow rectangles at random location until desired volume fraction is reached.

Table 1. Limiting conditions on two-point probability functions 


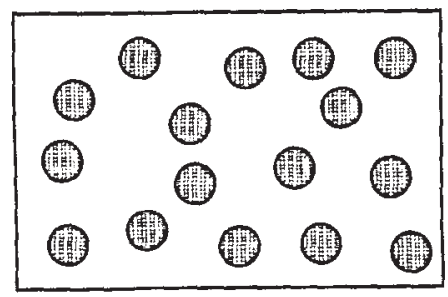

(a)

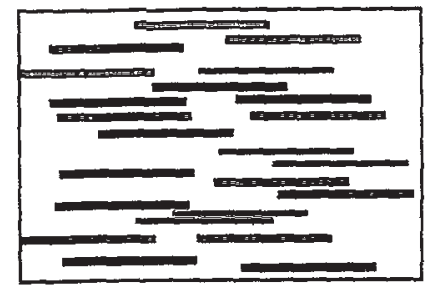

(b)

Fig. 3. Two kinds of two-isotropic-phase mediums.

The stresses and strain rates are normalized using $\sigma^{\prime}=\sigma / \sigma_{0}$ and $D^{\prime}=D / D_{0}$ for both phases in the medium. Such a normalization reduces the power law relation (eqn (1)) to:

$$
\frac{D^{\prime}}{D_{0}^{*}}=\left[\frac{\sigma^{\prime}}{\sigma_{0}^{*}}\right]^{n}
$$

where $D_{0}^{*}=D^{*} / D_{0}$ and $\sigma_{0}^{*}=\sigma^{*} / \sigma_{0}$. The normalized reference strain rate and stress are then unitless quantities and are $D_{0}^{*}=1$ for both phases, and $\sigma_{0}^{*}=1$ for phase 1 and $\sigma_{0}^{*}=0.33$ for phase 2 in both microstructures a and $b$. A power of $n_{1}$ and $n_{2}$ are chosen for the two phases. The volume fraction of phase 1 for microstructure a was $V_{1}=0.62$ and for microstructure $b V_{1}=0.74$.

Figures 4 and 5 show the results of the analysis to produce macroscopic stress-strain rate for the microstructures in Fig. 3. The parameters for the second phase material were chosen such that the microstructure behaves as a composite with the second phase as hard reinforcements. All simulations were performed to calculate the first order correction to the zeroth order term "Taylor's term". Further analysis results showed that the second

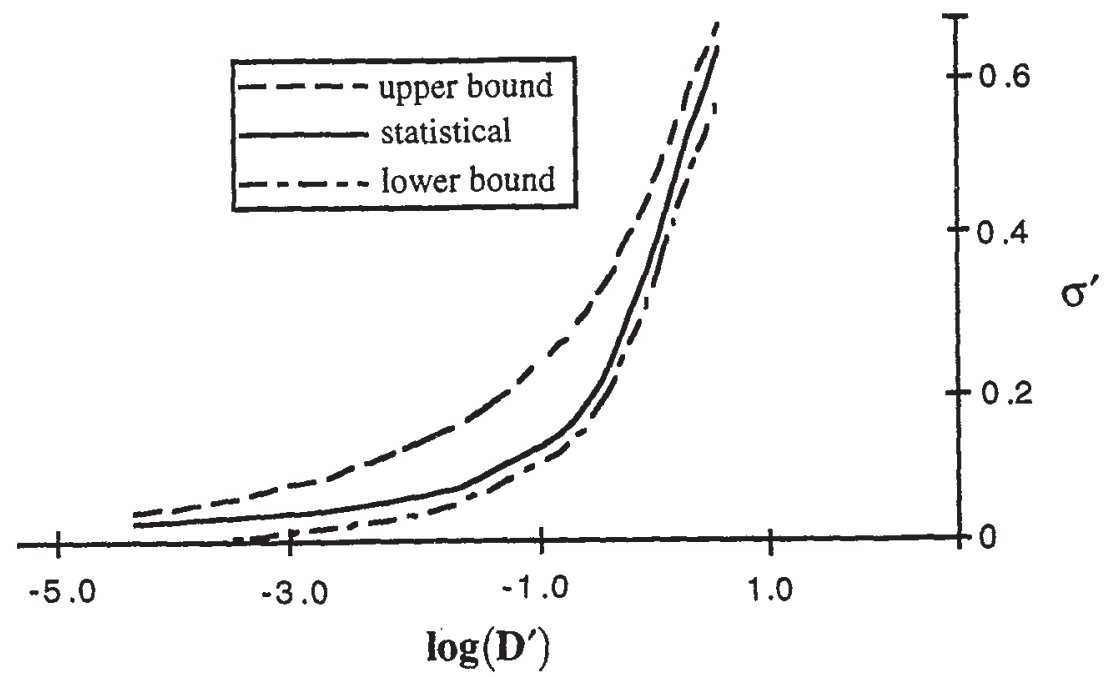

Fig. 4. Normalized macroscopic stress versus logarithm of normalized macroscopic strain rate for the microstructure a. 


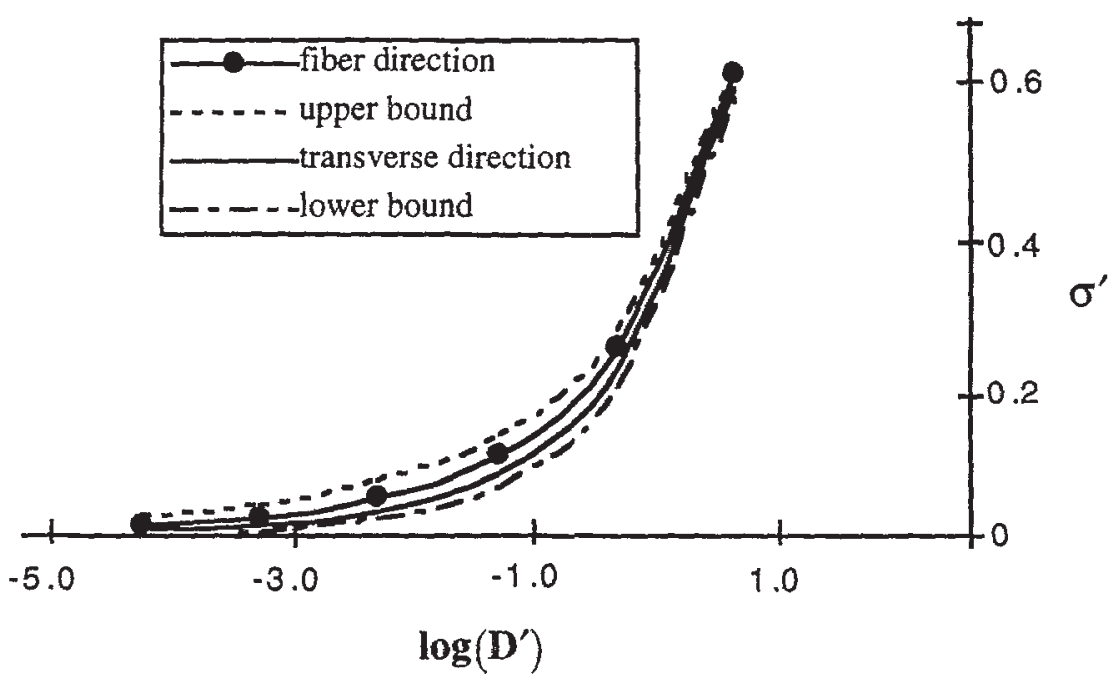

Fig. 5. Normalized macroscopic stress versus logarithm of normalized macroscopic strain rate for the microstructure b.

order term contribution. was negligible in the statistical formulation. In the case of the random structure (Fig. 3(a)) a maximum difference of $25 \%$ was observed when compared to the Taylor's upper bound. The Taylor's upper bound was simply the zeroth term (volume average) in the Taylor's expansion in eqn (41). For the anisotropic microstructure (Fig. 3(b)), the stress-strain rate results are plotted for the two cases where the stress is applied in the fiber and in the transverse directions. These results were then compared to the upper bound (Taylor's zeroth term) and the lower bound. The upper and lower bound calculation are analogous to Voigt (1889), and Reuss (1929) models. The lower bound is calculated by assuming that the stresses are the same in the two phases and the macroscopic strain-rate is the sum of $V_{1} L_{1}$ and $V_{2} L_{2}$, where $V_{1}$ and $V_{2}$ are volume fractions, $L_{1}$ and $L_{2}$ are strain-rates for the two phases, respectively. The results show that the material is harder (higher level of stress) once pulled in the fiber direction (Fig. 5).

\section{SUMMARY AND CONCLUSION}

This paper focuses on the application of the statistical continuum inelasticity theory to a medium consisting of two isotropic phases.

The simulation of the model presents results for two well known cases where in one case the microstructure is random and in the second case it is anisotropic. The statistical continuum model shows a maximum of $(25 \%)$ difference from Taylor's upper bound for the random microstructure. On the other hand the prediction for unidirectionally oriented narrow rectangular structures showed a drastic difference of $(66 \%)$ once the specimen was stressed in the fiber direction compared to the case when it was stressed in the transverse direction. This shows that incorporating second order statistics in the form of two point probability functions can predicted the true nature of anisotropy in heterogeneous microstructures. It is interesting to note that the Taylor's like models can lead to isotropy for such microstructure. 
It is found that the success of the theory depends on the existence of analytical equation for the probability functions. The probability functions for more complex cases requires more intensive work.

The present theory uses the secant-modulus form of the single-crystal constitutive law which is best suited to the problem of creep since the stress is small compared to the yield stress. For large stress and strain-rate problems, the tangent-modulus may be more suitable (Adams et al., 1989). The theory can only be applied to problems with small perturbations from the uniform strain-rate approximation of the Taylor's theory.

Acknowledgements-This work was supported in part (B. L. Adams) by the MRSEC Program of the National Science Foundation-under Award no. DMR-9632556. Further support was provided by the Center for Materials Research and Technology (MARTECH at FSU) and Center for Nonlinear and Nonequilibrium Sciences (CENNAS at FAMU).

\section{REFERENCES}

Adams, B. L, Canova, G. R. and Molinari, A. (1989) A statistical formulation of viscoplastic behavior in heterogeneous polycrystals. Textures and Microstructures 11, 57-71.

Ahzi, S. (1987) A self-consistent approach for the viscoplastic behavior of polycrystals under large deformations. Ph.D thesis, Universite de Metz, France.

Beran, M. J. (1968) Statistical Continuum Theories. Interscience, New York.

Beran, M. J., Mason, T. A., Adams, B. L. and Olsen, T. (1996) Bounding elastic constants of an orthotropio polycrystal using measurements of the microstructure. J. Mech. Phys. Solids 44, 1543-1563.

Bishop, J. F. W. and Hill, R. (1951) A theoretical derivation of the plastic properties of a polycrystalline facecentred. Phil. Mag. 42, 1298.

Corson, P. B. (1974a) Correlation functions for predicting properties of heterogeneous materials. I. Experimental measurement of spatial correlation functions in multiphase solids. J. Applied Physics, 45, 3159.

Corson, P. B. (1974b) Correlation functions for predicting properties of heterogeneous materials. II. Empirical construction of spatial correlation functions for two phase solids. J. Applied Physics, 45, 3165 .

Hashin, Z. and Shtrikman, S. (1962) A variational approach to the theory of the elastic behavior of polycrystals. J. Mech. Phys. Solids 10, 343.

Hill, R. (1952) The elastic behavior of a crystalline aggregate. Proc. Phys. Soc. A 65, 349.

Hill, R. (1965) Continuum micro-mechanics of elastoplastic polycrystals. J. Mech. Phys. Solids 13, 89.

Hill, R. (1967) The essential structure of constitutive laws for metal composites and polycrystals. J. Mech. Phys. Solids $15,79$.

Hutchinson, J. W. (1976) Bounds and self-consistent estimates for creep of polycrystalline materials. Proc Royal Society London, A. 348, 101.

Kroner, E. (1972) Statistical Continuum Mechanics. Springer-Verlag, Wien.

Kroner, E. (1987) Statistical Modelling In Modelling Small Deformation of Polycrystals, ed. J. Gittus, and J. Zarka, p. 229. Elsevier Applied Science, New York.

Miller, M. N. (1969) Bounds for effective bulk modulus of heterogeneous materials. J. Math. Phys. 10, 11.

Molinari, A., Canova, G. R. and Ahzi, S. (1987) A self consistent approach of the large deformation polycrystal viscoplasticity. Acta Metall. 35, 2983-2994.

Morris, P. R. (1970) Elastic constants of polycrystals. Int. J. Engeng. Sci. 8, 49-61.

Mura, T. (1987) Micromechanics of Defects in Solids. Kluwer Academic, Dordrecht, The Netherlands.

Reuss, A. (1929) Berechnung der Fliessgrenze von Mischkristallen auf Grund der Plastizitatsbedingung fur Einkristalle. Z. Angew. Math. Mech. 9, 49-58.

Taylor, G. I. (1938) Plastic strain in metals. J. Inst. Metals 62, 307.

Voigt, W. (1889) Uber die Beziehung zwischen den beiden Elastizitatskonstanten isotroper Korper. Wied. Ann. 38, 573-587. 